\title{
Hemoglobina $O$ arabia y hemoblobina Hofu: Reporte de tres familias en el Perú.
}

\author{
HAZAN Eliza*, NAVARRO Juan**, MARQUEZ María del Carmen***, \\ CASTILLO Jorge $* * * *$.
}

*Médico Jefe del Servicio de Hematología del Hospital Nacional Edgardo Rebagliati Martins del Instituto Peruano de Seguridad Social

**Médico Asistente del Servicio de Hematología. Clínica del Hospital Nacional Edgardo Rebagliati Martins del Instituto Peruano de Seguridad Social.

***Laboratorista clínico del Servicio de Hematología del Hospital Nacional Edgardo Rebagliati Martins del Instituto Peruano de Seguridad Social.

****Médico Jefe del Departamento de Onco-Hematología del Hospital Nacional Edgardo Rebagliati Martins del Instituto Peruano de Seguridad Social.

\section{SUMMARY}

We report the finding of two abnormal hemoglobins in three Peruvian families: hemaglobin $O$ Arab and Hemoglobin Hofu. In the first case the patient was found to be double heterozygous for hemoglobin $O \mathrm{Arab} / \boldsymbol{\beta}$ thalassaemia and presents a moderate chronic hemolytic anemia. In the second family the propositus had a severe chronic of hemoglobin A/O Arab were asymptomatic. In the third case we report a family with a very mild chronic hemolytic anemia due to the presence of hemoglobyn Hofu. These two abnormal hemoglobin O Arabia was introduced in Peru and described the methodology used in the Laboratory to characterize the abnormal hemoglobins.

KEY WORDS: Hb O Arab, Hb Hofu, hemolytic anemia, abnormal haemoglobin.

\section{INTRODUCCIÓN}

En el Perú relativamente pocos trabajos sobre hemoglobinas anormales han sido publicados a pesar de la gran variedad de grupos étnicos que conforman el país.

Aste fué el primero que realizó estudios de variaciones genéticas de la hemoglobina en población negra, encontrando una significante prevalencia de rasgo de hemoglobina $\mathrm{S}$ y algunos casos de hemoglobina $\mathrm{C}$ en Chincha, pueblo ubicado al Sur de Lima (1).

Posteriormente Jerí y cols, reportan la presencia de una variedad rara de alfa talasemia intermedia conocida como enfermedad por hemoglobina $\mathrm{H}$ en dos familias peruanas de ancestro chino (2); Castillo en despistaje realizado en la ciudad de Lima en mil personas, todos ellos peruanos de origen chino, encuentra una muy baja prevalencia de talasemia menor (3). 
El presente reporte describe dos variedades de hemoglobina encontradas por primera vez en el Perú; una es la hemoglobina O Arabia, descrita originalmente por Ramot y Cols, en Israel al encontrarla en un niño árabe y su hermana (4), y la hemoglobina Hofu, una hemoglobina de muy rara ocurrencia de la cual probablemente no existan muchos casos en el mundo, reportada por primera vez en Japón en 1968 (5) y encontrada en nuestro país en una paciente con cuadro de leve anemia crónica asintomática.

\section{$\operatorname{Caso} \mathbf{N}^{0} 1$}

F.A.F., varón de 67 años, mestizo, natural de la zona norte del Perú, fue hospitalizado en Junio de 1984 debido a dolor abdominal, anemia, ictericia y dolores óseos difusos. En repetidas ocasiones había sido internado en nosocomios de su región con síntomas parecidos habiéndose llegado al diagnóstico de anemia hemolítica crónica hereditaria. El examen físico reveló además de palidez y moderada ictericia marcada hepatoesplenomegalia. Ambos padres, ya fallecidos, eran originarios de Perú.

\section{Exámenes de Laboratorio}

Los valores hematológicos están consignados en el cuadro $\mathrm{N}^{\circ} 1$ el cual incluye también a la familia estudiada. Exámenes bioquímicos de rutina se encontraron dentro de límites normales excepto la bilirrubina que estuvo elevada a predominio de la fracción no conjugada. El test Coombs directo fué negativo, el hierro sérico 144/ug/dl(vn: 60-160 ug/dl), la capacidad de combinación de la transferrina $250 \mathrm{ug} / \mathrm{dl}$ (vn: 280-400 ug/dl) y la saturación 58\% (vn: 20-60\%). El frontis de sangre periféricas mostró importante hipocromía, numerosas células en diana y presencia de ortocromáticos. El examen de la médula ósea reveló marcada hiperplasia de la serie eritroide con incremento de hemosiderina. La electroforesis de hemoglobina en acetato de celulosa a $\mathrm{pH}$ alcalino hizo visible una banda único que migraña como hemoglobina $\mathrm{C}$ (Figura $\mathrm{N}^{\circ} 1$ ). La electroforesis de hemoglobina en agar citrato a $\mathrm{pH}$ ácido demostró una banda que migraba entre la hemoglobina $\mathrm{A}$ y la hemoglobina $\mathrm{S}$ y una pequeña banda de hemoglobina fetal. La electroforesis de globina a $\mathrm{pH}$ alcalino evidenció ausencia de la cadena $\beta$ A y presencia de una banda $\beta$ situada entre la cadena $\beta$ S y la cadena $\alpha$ (Figura №2). La electroforesis de globina a $\mathrm{pH}$ ácido mostró una banda que migraba como la cadena $\beta \mathrm{S}$ además de la cadena $\alpha$ ( $\left.\underline{\text { Figura } \mathrm{N}^{\circ} 3}\right)$.

La movilidad electroforética de esta hemoglobina mutante fué compatible con la hemoglobina $\mathrm{O}$ Arabia lo cual fué corroborado al enviar muestra de sangre a centros de referencia del exterior.

La ausencia de hemoglobina $\mathrm{A}$ en la electroforesis en acetato de celulosa a $\mathrm{pH}$ alcalino en el paciente, asociada a los hallazgos electroforéticos de $\beta$ talasemia menor en sus hijos nos confirmó además que era portador de $\beta^{\circ}$ talasemia.

\section{Estudio Familiar}

Fueron estudiados además del paciente, sus seis hijos, encontrándose que tres de ellos eran heterocigotos para hemoglobina A/O Arabia y los otros tres portadores de $\beta$ talasemia menor. La esposa mostraba hemoglobina A/A (Figura $\mathrm{N}^{\circ} 4$ ).

\section{Caso $\mathbf{N}^{\circ} 2$}


M.M.B. niña de cinco años de edad, proviene de la misma zona geográfica que el paciente del caso $\mathrm{N}^{\mathrm{o}} 1$ aunque aparentemente no relacionados, ingresa al hospital para ser evaluada por anemia, infecciones frecuentes, artralgias, hiporexia y fiebre. Como antecedentes registra haber recibido transfusiones de sangre en doce oportunidades así como tratamiento antianémico a base de hierro y multivitamínicos sin éxito alguno. No existen antecedentes de anemia en la familia. Al examen físico la paciente luce pálida, adelgazada y con pobre desarrollo físico. Se detecta soplo sistólico funcional y marcado hepato-esplenomegalia.

\section{Exámenes de Laboratorio:}

Los valores hematológicos de la paciente y sus padres se presentan en el cuadro $\mathrm{N}^{\mathrm{0}} 2$. Las pruebas bioquímicas de rutina fueron encontradas normales con excepción de la deshidrogenasa láctica la cual mostró niveles muy elevados. El frotis de sangre periférica mostró hipocromía, células en diana y drepanocitos.

El estudio de la médula ósea reveló marcada hiperplasia eritroide normoblástica con algunos megaloblastos intermedios. La electroforesis de hemoglobina en acetato de celulosa a $\mathrm{pH}$ alcalino mostró la presencia de dos bandas, una que migraba con hemoglobina $\mathrm{S}$ y otra más lenta que se ubicaba donde usualmente lo hace la hemoglobina $\mathrm{C}$ (Figura $\mathrm{N}^{\circ}$ ) $)$. La electroforesis en agar citrato a $\mathrm{pH}$ ácido demostró igualmente dos bandas, una que migraba entre la hemoglobina $\mathrm{A}$ y la hemoglobina $\mathrm{s}$ y otra que lo hacía como hemoglobina $\mathrm{S}$. La electroforesis de globina a $\mathrm{pH}$ ácido hizo visible una cadena $\alpha$ y una única cadena $\beta$ en posición beta $\mathrm{S}$. La prueba de solubilidad fué positiva. Todos los corridos electroforéticos fueron realizados contra un patrón conocido de hemoglobina O Arabia.

\section{Estudio familiar}

Con la paciente fueron estudiados los padres y un hermano, encontrándose que el padre tenía rasgos falcémico y la madre era heterocigote a hemoglobina $\mathrm{O}$ Arabia. Su único hermano resultó hematológicamente normal. (Figura $\mathrm{N}^{\mathrm{0}} 6$ ).

\section{Caso $\mathbf{N}^{\circ} 3$}

B.L.M., mujer de 35 años, con rasgos asiáticos, hija de padre de origen chino y madre de ascendencia italiana acude a nuestro Servicio de Hematología por presentar anemia crónica. A lo largo de su vida había recibido en varias oportunidades tratamiento con fierro oral sin aparente mejora. El examen clínico reveló a una paciente en aparente buen estado general, asintomática y sin evidencia de visceromegalia.

\section{Exámenes de Laboratorio:}

Los valores hematológicos de la paciente incluyendo los encontrados en el resto de la familia estudiada se hallan sumarizados en el cuadro $\mathrm{N}^{\circ} 3$. Los valores bioquímicos de rutina se encontraron todos dentro de límites normales. En el examen del frotis de sangre periférica se observó sólo moderada hipocromía. El estudio de la médula ósea mostró moderada hiperplasia eritroide con depósito de hierro conservado. La electroforesis de hemoglobina en acetato de celulosa a $\mathrm{pH}$ alcalino reveló la presencia 
de dos bandas características, una que migraba como hemoglobina A adulta y otra que lo hacía más anódicamente que ésta (Figura $\mathrm{N}^{\circ} 7$ ). El patrón electroforético en citrato agar a $\mathrm{pH}$ ácido fué normal $\mathrm{A} / \mathrm{A}$. La electroforesis de globina tanto a $\mathrm{pH}$ ácido como alcalino hizo evidente una banda extra de cadena $\beta$ de rápida migración. La cuantificación de la hemoglobina fetal fué de $2 \%$ y la inducción de cuerpos de inclusión intra eritrocitario fué positiva a la 48 horas de incubación con azul de cresil brillante. El Laboratorio de Biología Molecular del Center for Disease Control de Atlanta, Georgia, logró demostrar que la variante en estudio era la hemoglobina Hofu.

\section{Estudio familiar}

Se estudió la familia de la paciente encontrándose la misma variante de hemoglobina en las dos hermanas y en la madre. El padre había fallecido años atrás. (igura No8).

\section{DISCUSION}

La hemoglobina $\mathrm{O}$ es una hemoglobina anormal relativamente rara, descrita originalmente en Indonesia en 1958 (9). Posteriormente una hemoglobina con similares características electroforéticas fue encontrada en un niño árabe y su hermana en Israel en 1960 y reportada por Ramot y co. (4); ambos tipos de hemoglobina fueron encontrados diferentes desde el punto de vista estructural en 1962 por Baglioni y Lehman quienes pudieron precisar que la hemoglobina $\mathrm{O}$ descrita en la Indonesia correspondía a una variante de la cadena $\alpha$ mientras que la descrita en al familia árabe representaba una alteración de la cadena $\beta$ (10).

Los casos que reportamos como hemoglobina $\mathrm{O}$ corresponden a la variante descrita por Ramot y las consideramos hemoglobina O Arabia, la cual es resultante de la sustitución de ácido glutámico por lisina en la posición 121 de la cadena beta. En su forma heterocigote la presencia de la hemoglobina $\mathrm{O}$ Arabia, puede condicionar una muy moderada anemia y escasos cambios morfológicos de los eritrocitos $(11,12)$, con cierta frecuencia se encuentra en estado doble heterocigoto con la hemoglobina $S(13,14,15$; con $\beta^{\mathrm{o}}$ y $\beta+$ talasemias $(16,17,18,19,20,21)$ y con la hemoglobina $C(14,15,22)$, produciendo un cuadro de anemia hemolítica, de moderada a severa intensidad en combinación con las hemoglobinas $\mathrm{S}, \mathrm{C}, \mathrm{y} \beta^{\mathrm{o}}$ talasemia y muy leve en el caso que coexista con $\beta+$ talasemia $(16,17,18,19,20,21)$ y con la hemoglobina $C(14,15,22)$, produciendo un cuadro de anemia hemolítica, de moderada a severa intensidad en combinación con las hemoglobinas $\mathrm{S}, \mathrm{C}, \mathrm{y} \beta^{\mathrm{o}}$ talasemia y muy leve en el caso que coexista con $\beta+$ talasemia.

Los cuadros clínicos de nuestros casos 1 y 2 son acordes con lo descrito anteriormente, aunque es muy probable que la paciente con la combinación S/O Arabia, dada su corta edad y lo agresivo de la evolución clínica, pueda seguir el curso descriptivo en otras publicaciones $(23,24)$. El hecho que la hemoglobina $\mathrm{O}$ Arabia migre igual que la hemoglobina $\mathrm{C}$ en la electroforesis en acetato de celulosa a $\mathrm{pH}$ alcalino ocasiona que casos de hemoglobina S/O Arabia puedan ser confundidos con aquellos de hemoglobina $\mathrm{S} / \mathrm{C}$, más aún cuando el cuadro clínico en ambas situaciones es parecido. Esto puede inducir errores en cuanto a apreciación de variantes de hemoglobina en determinadas zonas lo cual podría obviarse si a todos los posibles casos de hemoglobina $\mathrm{S} / \mathrm{C}$ se les hiciera electroforesis en citrato agar a $\mathrm{pH}$ ácido (25). 
La distribución geográfica de la hemoglobina O Arabia es variada; ha sido encontrada en Saudi Arabia (20), Sudán (13)(26), Yemén (20), Bulgaria (16,17), Egipto (27), Grecia (22), Jamaica (15), Norteamerica $(12,14,28)$ y población turca en Chipre (29).

Algunos consideran que esta hemoglobina podría ser utilizada como marcador antropológico para explicar movilización de poblaciones del Medio Oriente y Africa hacia Europa y posteriormente el resto del mundo (17,27). Probablemente llega al Perú con la inmigración de pobladores de Africa, negros en su mayoría, traídos a nuestro país a fines del siglo pasado para ocuparse en labores de campo. La posterior integración de estos grupos humanos quienes trajeron la hemoglobina $\mathrm{S}$ y la hemoglobina $\mathrm{C}$ con gente propia de la región, indios en su mayoría, contribuyó a que esta hemoglobina se haya encontrado en personas de raza mestiza como la que reportamos en el presente trabajo.

Creemos que la presencia de esta hemoglobina en América del Sur puede ser más frecuente que lo conocido hasta la fecha, la poca expresividad clínica y hematológica del rasgo de la hemoglobina $\mathrm{O}$ Arabia hacen difícil su detención; sólo con surveys regionales podríamos tener una apreciación más concreta de la verdadera prevalencia de esta hemoglobina en nuestro medio.

La hemoglobina Hofu fué descrita por Miyaji y col. En 1968 en Japón cuando hacían estudios poblacionales de variantes de hemoglobina en zonas alrededor de la ciudad de Hofu (5). Fué encontrada en una paciente de 55 años con síntomas de gastritis a quien se le encontró además una leve anemia estudio electroforético de la hemoglobina realizado en gel de agar a $\mathrm{pH} 8.6$ demostró la presencia de una banda que se situaba más hacia el ánodo que la hemoglobina A adulta pero era inseparable de ella a pH neutro. Estudios estructurales de la misma revelaron que es una hemoglobina en la cual la valina de la cadena $\beta$ en la posición 126 es substituida por ácido glutámico ( $\beta 126$ valglu).

La hemoglobina Hofu parece ser muy rara, característicamente en su forma heterocigota no va asociada a síntomas. Reportada originalmente en Japón en 1968 y posteriormente en varios casos más en ese mismo país (30,31), ha sido también encontrada en España en donde se han descritos más de 11 casos (32).

En Latinoamérica fué reportada por Arends en una paciente proveniente de Sevilla en quien se encontró hemoglobína Hofu asociada a $\beta^{\circ}$ talasemia (33).

En el caso que describimos, la presencia de esta hemoglobina prácticamente no ocasiona síntomas en el a propósito ni en sus hermanas. Es interesante observar que el haber encontrado esta hemoglobina en una paciente con mestizaje asiático e italiano, la anormalidad encontrada en forma heterocigótica probablemente proviene de la parte europea, a pesar de no ser una anormalidad descrita con excepción del caso de Arends, en Europa meridional. Al momento creemos difícil, dado lo raro de esta hemoglobina, tratar de proponer una hipótesis en movimientos poblacionales para explicar su aparición en nuestro país.

Posiblemente la posterior descripción de mayor número de casos contribuya a aclarar nuestra interrogante. 


\section{REFERENCIAS BIBLIOGRÁFICAS}

1. Aste H. Diferenciación de hemoglobinas humanas. En: Concytec. Cuadernos Científicos y Tecnológicos. Lima: Concytec. 1984; 2: 61-64.

2. Jerí A, Rojas M, Castillo J. Enfermedad por Hemoglobina H. Estudio de dos familias peruanas. Sangre 1976; 21: 67-76.

3. Castillo AJ. Estudio electroforético de hemoglobinas en 1000 personas de origen chino en la ciudad de Lima. III Congreso Peruano de Hematología; Lima, 1980.

4. Ramot B, Fuischer S, Remez D, Scheneerson R, Kahane D, Ager J. Hemoglobin O In a Araba family: Sickle cell Hb O trait. Brit Med J 1960; 2: 1262-1264.

5. Miyaji T, Ohba Y, Yamamoto K, Shibata S, Iuchi I, Takenaka M. Japanese Hemoglobin Variant. Nature 1968; 217: 89-90.

6. Dacie JV, Lewis SM. Basic haematologic techniques in practical haematology. Churchill Livingstone, London 1975; 21-68.

7. Sáenz Renauld G, Moreira Pereira J. Laboratorio Hemoglobinopatías. Manual Latinoamericano. Ministerio de Salud. Costa Rica. 1980.

8. International Committee for Standardization in Hematology. Recommendations of a System for Identifying Abnormal Hemoglobin. Blood 1978; 52: 1065-1067.

9. Lie-Injo Luan Eng and Sadono. Hemoglobin O (Buginese X) in Sulawesi. Brit Med J 1958; 1: 1461-1462.

10. Baglioni C, Lehman H. Chemical Heterogeneity of Hemoglobin O. Nature 1962; 196: 229-232.

11. Tcholokav B, Kancev KN. New cases o Hemoglobinopathy O Arab in the District of Burgas. Vatr bol 1974; 13: 51-54.

12. Schneider RG, Haggard ME, Gustavson LP, Brimhall B, Jones RT. Genetic haemoglobinn abnormalities in about 9000 black and 7000 white newborns; Haemoglobin F Dickinson, a new variant. Brit J Haemat 1974; 28: 515-524.

13. Ibrahim SA, Mustafa D. Sickle-cell Haemoglobin o Disease in Sudanese family. Brit Med J 1967; 3: 715-717.

14. Maeda K, Kini R, Saeed S, Ruchnagel D. Hemoglobin S O Arab and Hemoglobin C O Arab diseases. Am J Ped Hemat Oncol 1983; 5: 127-131.

15. Milner P, Miller C, Grey R, Seakins M, DeJoong W, Went LN. Hemoglobin O Arab in four negro families and its interaction with hemoglobin S and Hemoglobin C. N Engl J Med 1970; 283: 1417-1425.

16. Kantchev NK, Tcholakov B, Baglioni C, Colombo B. Hemoglobin O Arab in Bulgaria. Nature 1965; 205: 187-188.

17. Kantchev $\mathrm{KN}<$ Tcholakov B, Casey R, Lehman H, el Hazmi M. Twelve families with O Arab in the Burgas District of Bulgaria. Observations on Sixteen Examples of $\mathrm{Hb} \mathrm{O}$ Arab-B ${ }^{\circ}$ thalassaemia. Humagenetik 1975; 26: 93-97.

18. Audhuy B Nort ML, Pinget M, Galacteros F, Mayer S, dans une familie algerienne. Nouv Rev Fr Hematol 1983 ; 24 : 289-294.

19. Morle F, Morle L, Baklouti $\mathrm{F}$ et al. The $\mathrm{Hb} \mathrm{F}$ composition in a Moroccan family with $\mathrm{B}^{\mathbf{0}}$ Thalassemia and $\mathrm{Hb} \mathrm{O}-A r a b$. Scand J Haematol 1984; 33: 281-287.

20. El Hazmi MAF, Lehman H. Human haemoglobins and haemoglobinapathies in Arabia: Hb O Arab in Saudi Arabia. Acta Haemat 1980; 63: 268-273.

21. Penev MN, Panova DY, Yvanov BN. Three cases of Hemogobin O Arab. Folia Haematol 1977; 104: 271-276. 
22. Sharma RS, Williams SL, Baptist NG, Fischer WK, Thompson OP. Haemoglobin $\mathrm{C}$ and Haemoglobin O Arab-Thalassaemia in families of Greek origin. Pathology 1976; 8: 89-93.

23. Klingersmith WC, Danich EH, Dover GJ, Wagner HN. Delineation of paripheral bone infarcts in a child with a rare Hemoglobinopathy ( $\mathrm{S} O \mathrm{O}$ Arab) and purpura fulminans: Case report. J Nucl Med 1976; 17: 1062-1064.

24. Gilman PA, Abel AS. Acute splenic sequestration in Hemoglobin sickle O-Arab disease. Johns Hopkins Med J 1980; 146: 285-288.

25. David J. Hemoglobin S O Arabia disease in a black American. Am J Med Sci 1973; 267-274.

26. Vella F, Beale D, Lehman H. Haemoglobin O Arab in Sudance. Nature 1966; 209: 308-309.

27. Barakat A, Hemoglobin O Arab in Egypt and Aden: Possible errors resulting from the use of hemoglobins variants as genetic markers in populations surveys. Z Morphol Anthropol 1967; 59: 100-103.

28. Atwater J, Ragan J. Hemoglobin O Arab trait in three generations of an American negro family. Abs $10^{\text {th }}$ Cong Int Soc Hematol Stockholm, 1964.

29. Cin S, Akar N, Arcasoy A, Cavdar A, Dedeoglu S. Haemoglobin O Arab (B 121 Gly-Lys) in Turkish Cypriot population. J Med Gen (1984; 21: 158.

30. Ohba Y, Matsouka M, Fuyuno K, Nishijima S, Miyaji T. Further studies on Hb Hofu, beta $126(\mathrm{H} 4)$ Val replaced by Glu with special reference to is stability. Hemoglobin 1981; 5(1): 89-95.

31. Yokota E, Sugihara J, Kagimoto M, Naito E, Matsuo T, Imamura T, Yamada H, Imoto T. Hemoglobin Hofu: beta 126 (H4) valine - glutamic acid observed in a Japanese Family. Hemoglobin 1984; 8(6): 627-30.

32. Martin G, Villegas A, Calero F, Porres A, Espinos D. Two new families with Hemoglobin Hofu discovered in Spain. Hematologica - Pavia 1988; 73 (2): 115-8.

33. Arends T, Garlin G, Guevara JM, Amesty C, Pérez - Bandez O, Lorkin PA, Lehman H, Castillo O. Hemoglobin Hofu associated with beta O Thalassemia. Acta Hematol - Basel 1985; 73(1): 51-4.

\section{Correspondencia:}

Eliza Hazán de Heraud

Parque Naciones Unidas 104/203.

Miraflores Lima 18- PERU 